Supplementary information for the paper:

J. Chem. Eng. Data, 2006

\title{
Thermochemistry of Chlorobenzenes and Chlorophenols: Ambient Temperature Vapor Pressures and Enthalpies of Phase Transitions. Experiment and Calculations.
}

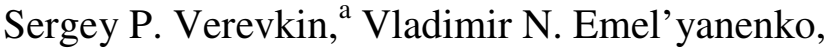

Department of Physical Chemistry, University of Rostock, 18051 Rostock, Germany

Andreas Klamt

COSMOlogic GmbH\&CoKG, Burscheider Str. 515, 51381 Leverkusen, Germany

and Institute of Physical Chemistry, University of Regensburg, 93040 Regensburg

a To whom correspondence should be addressed, e-mail: sergey.verevkin@uni-rostock.de.

Phone: +49-381-498-6508. FAX: +49-381-498-6502 
Matrix forCalculation of Enthalpies of Vaporization $\Delta_{1}^{\mathrm{g}} \mathrm{H}_{\mathrm{m}}$ of Chlorobenzenes and Chlorophenols at 298.15 K

\begin{tabular}{|c|c|c|c|c|c|c|c|c|c|c|c|c|c|}
\hline Соединение & $\begin{array}{l}\text { ortho } \\
\mathrm{Cl}-\mathrm{Cl}\end{array}$ & $\begin{array}{l}\text { para } \\
\mathrm{Cl}-\mathrm{Cl}\end{array}$ & $\begin{array}{l}\text { meta } \\
\mathrm{Cl}-\mathrm{Cl}\end{array}$ & $\begin{array}{l}\text { ortho } \\
\text { OH-Cl } \\
(\mathrm{HB})\end{array}$ & $\begin{array}{c}\text { para } \\
\mathrm{OH}-\mathrm{Cl}\end{array}$ & $\begin{array}{l}\text { meta } \\
\mathrm{OH}-\mathrm{Cl}\end{array}$ & $\begin{array}{l}\text { ortho } \mathrm{OH}-\mathrm{Cl} \\
\quad(\text { nonHB) }\end{array}$ & $\Delta \mathrm{H}(H \rightarrow O H)$ & $\Delta \mathrm{H}(H \rightarrow C l)$ & exp. & calc. & exp-calc & ABS \\
\hline 1,2-dichlorobenzene & 1 & 0 & 0 & 0 & 0 & 0 & 0 & 0 & 2 & 48.82 & 49.06 & -0.24 & 0.24 \\
\hline 1,3-dichlorobenzene & 0 & 0 & 1 & 0 & 0 & 0 & 0 & 0 & 2 & 47.68 & 48.24 & -0.56 & 0.56 \\
\hline 1,4-dichlorobenzene & 0 & 1 & 0 & 0 & 0 & 0 & 0 & 0 & 2 & 47.60 & 47.99 & -0.39 & 0.39 \\
\hline 1,2,3-trichlorobenzene & 2 & 0 & 1 & 0 & 0 & 0 & 0 & 0 & 3 & 57.55 & 56.96 & 0.59 & 0.59 \\
\hline 1,2,4-trichlorobenzene & 1 & 1 & 1 & 0 & 0 & 0 & 0 & 0 & 3 & 55.30 & 55.88 & -0.58 & 0.58 \\
\hline 1,3,5-trichlorobenzene & 0 & 0 & 3 & 0 & 0 & 0 & 0 & 0 & 3 & 55.56 & 55.31 & 0.25 & 0.25 \\
\hline 1,2,3,4-tetrachlorobenzene & 3 & 1 & 2 & 0 & 0 & 0 & 0 & 0 & 4 & 62.70 & 64.44 & -1.74 & 1.74 \\
\hline 1,2,4,5-tetrachlorobenzene & 2 & 2 & 2 & 0 & 0 & 0 & 0 & 0 & 4 & 63.04 & 63.37 & -0.33 & 0.33 \\
\hline 1,2,4,6-tetrachlorobenzene & 2 & 1 & 3 & 0 & 0 & 0 & 0 & 0 & 4 & 62.30 & 63.62 & -1.32 & 1.32 \\
\hline pentachlorobenzene & 4 & 2 & 4 & 0 & 0 & 0 & 0 & 0 & 5 & 70.14 & 71.76 & -1.62 & 1.62 \\
\hline hexachlorobenzene & 6 & 3 & 6 & 0 & 0 & 0 & 0 & 0 & 6 & 81.20 & 79.75 & 1.45 & 1.45 \\
\hline 2-chlorophenol & 0 & 0 & 0 & 1 & 0 & 0 & 0 & 1 & 1 & 52.34 & 51.18 & 1.16 & 1.16 \\
\hline 3-chlorophenol & 0 & 0 & 0 & 0 & 0 & 1 & 0 & 1 & 1 & 63.52 & 64.45 & -0.93 & 0.93 \\
\hline 4-chlorophenol & 0 & 0 & 0 & 0 & 1 & 0 & 0 & 1 & 1 & 64.42 & 65.04 & -0.62 & 0.62 \\
\hline 2,3-dichlorophenol & 1 & 0 & 0 & 1 & 0 & 1 & 0 & 1 & 2 & 57.34 & 58.49 & -1.15 & 1.15 \\
\hline 2,4-dichlorophenol & 0 & 0 & 1 & 1 & 1 & 0 & 0 & 1 & 2 & 58.97 & 58.26 & 0.71 & 0.71 \\
\hline 2,5-dichlorophenol & 0 & 1 & 0 & 1 & 0 & 1 & 0 & 1 & 2 & 56.69 & 57.41 & -0.72 & 0.72 \\
\hline 2,6-dichlorophenol & 0 & 0 & 1 & 1 & 0 & 0 & 1 & 1 & 2 & 59.58 & 60.99 & -1.41 & 1.41 \\
\hline 3,4-dichlorophenol & 1 & 0 & 0 & 0 & 1 & 1 & 0 & 1 & 2 & 70.84 & 72.35 & -1.51 & 1.51 \\
\hline 2,4,6-trichlorophenol & 0 & 0 & 3 & 1 & 1 & 0 & 1 & 1 & 3 & 67.16 & 67.90 & -0.74 & 0.74 \\
\hline pentachlorophenol & 4 & 2 & 4 & 1 & 1 & 2 & 1 & 1 & 5 & 85.00 & 82.85 & 2.15 & 2.15 \\
\hline $\mathrm{GAV}, \mathrm{kJ} / \mathrm{mol}$ & 0.66 & -0.41 & -0.16 & -14.02 & -0.16 & -0.75 & 2.57 & 24.2 & 7.4 & & & $\begin{array}{c}\text { Average } \\
\text { error }\end{array}$ & 0.70 \\
\hline
\end{tabular}

